1 Fundação Oswaldo Cruz (Fiocruz), Instituto Nacional de Saúde da Mulher, da Criança e do Adolescente Fernandes Figueira (IFF) - Rio de Janeiro (RJ), Brasil. marcialuiz@iff.fiocruz.br

2 Fundação Oswaldo Cruz (Fiocruz), Instituto Nacional de Saúde da Mulher, da Criança e do Adolescente Fernandes Figueira (IFF) - Rio de Janeiro (RJ), Brasil. andrezaenfermeira@gmail. com

3 Fundação Oswaldo Cruz (Fiocruz), Instituto Nacional de Saúde da Mulher, da Criança e do Adolescente Fernandes Figueira (IFF) - Rio de Janeiro (RJ), Brasil. cbonan@iff.fiocruz.br

\section{Planejamento reprodutivo na clínica da família de um Teias: condições facilitadoras e limites à assistência}

\author{
Reproductive planning in a family clinic within a Teias: facilitating \\ conditions and limits on assistance
}

Marcia dos Santos Luiz', Andreza Rodrigues Nakano², Claudia Bonan³
RESUMO Apresenta-se um estudo de caso com abordagem qualitativa que analisou a assistência ao planejamento reprodutivo de uma clínica da família inserida em um Território Integrado de Atenção à Saúde e que utilizou como referência teórico-analítica a continuidade, a coordenação e integração de cuidados em saúde reprodutiva e o potencial da assistência na promoção de direitos. A conjugação da Estratégia Saúde da Família com o Território Integrado de Atenção à Saúde apresentou avanços na assistência ao planejamento reprodutivo no que tange à organização de aspectos de infraestrutura e provisão de insumos, entretanto os avanços alcançados não foram suficientes para promover uma assistência que garanta direitos.

PALAVRAS-CHAVE Planejamento familiar; Atenção Primária à Saúde; Saúde da família; Avaliação em saúde.

ABSTRACT This article discusses the enabling conditions and limits on reproductive planning assistance in a clinical family in a Território Integrado de Atenção à Saúde. The theoretical and analytical framework included the continuity, coordination and integration of reproductive health care and the potential for assistance in promoting rights. The combination of Family Health Strategy and Território Integrado de Atenção à Saúde showed advances in assisting the reproductive planning assistance aspects of the organization of infrastructure and provision of inputs, however, presenting limits in promoting a service that guarantees rights.

KEYWORDS Family planning; Primary Health Care; Family health; Health evaluation. 


\section{Introdução}

A atenção à saúde sexual e reprodutiva é uma das áreas de atuação prioritárias da Atenção Básica (BRASIL, 2010), e sua oferta deve ter como princípio a garantia dos direitos sexuais e dos direitos reprodutivos, conforme inscritos nas políticas que vêm sendo desenvolvidas pelo Estado brasileiro, como a Política Nacional de Assistência Integral à Saúde da Mulher (BRASIL, 2004) e a Política Nacional de Direitos Sexuais e Reprodutivos (BRASIL, 2005). Inserido nesse nível da atenção, a assistência ao planejamento reprodutivo consiste, sobretudo, em ações de promoção, prevenção, informação e educação em saúde.

Estudos sobre a assistência ao planejamento reprodutivo no Brasil têm identificado problemas persistentes que vão desde o acesso aos serviços e aos insumos até o modelo de atenção, pouco favorável à promoção da equidade, da autonomia e dos direitos (MOURA; SILVA, 2006; COSTA; GUILHEM; SILVER, 2006; OSIS ET AL., 2006; HEILBORN ET AL., 2009; BONAN ET AL., 2010). A implantação de políticas de direitos reprodutivos e, particularmente, de planejamento reprodutivo tem sido um processo não linear, tendo em vista que a temática que entrecruza sexualidade, reprodução e direitos é objeto de acirradas disputas políticas e ideológicas. Entretanto, as dificuldades da assistência ao planejamento reprodutivo devem ser examinadas também à luz dos desafios impostos à própria Atenção Básica.

Criado em 1994 e ampliado com a Política Nacional da Atenção Básica, em 2006, o Programa Saúde da Família é uma importante estratégia para reorientação do modelo assistencial, propondo o fortalecimento da Atenção Básica como porta de entrada na rede de saúde e elemento-chave para a prestação de uma assistência integral. Entretanto, para que a Atenção Básica cumpra tal papel, é necessário avançar na concretização dos princípios diretivos do sistema de saúde, quais sejam: continuidade/ longitudinalidade, coordenação e integração de cuidados (STARFIELD, 2002; GIOVAnella et al., 2009; CUNHA; GIOVANELLA, 2011). A continuidade/ longitudinalidade do cuidado diz respeito à responsabilidade do serviço de saúde pela população adscrita ao longo do tempo, independentemente da presença de morbidade e da procura do usuário pelo serviço de saúde. O princípio da integração refere-se a uma atenção que integra ações de promoção, prevenção, assistência e reabilitação, promovendo acesso aos diferentes níveis de assistência e ofertando respostas ao conjunto de necessidades de saúde de uma comunidade, e não apenas a um recorte de problemas. $\mathrm{O}$ princípio da coordenação implica em uma atenção de modo comprometido em atender às demandas de saúde e prestar atenção coordenada à pessoa/família, seja entre ações ofertadas no próprio serviço ou mesmo nos casos de encaminhamento a outros níveis de atenção (BRASIL, 2010).

Nesse intuito, tem-se desenvolvido experiências de organização da prestação dos cuidados, como os Territórios Integrados de Atenção à Saúde (Teias), utilizando abordagens ecossistêmicas que articulam as dimensões biopsíquicas, políticas, ambientais, socioeconômicas e culturais da saúde e das práticas de saúde (ENGSTRON; FONSECA; LEIMANN, 2012). A proposta do Teias tem o potencial de conjugar duas questões que são de grande importância para a qualidade da assistência ao planejamento reprodutivo, na perspectiva da integralidade e da promoção dos direitos à saúde e dos direitos sexuais e reprodutivos: potencializar a integração, coordenação e continuidade dos cuidados; e, com as metodologias de abordagem ecossistêmica, enfrentar as desigualdades sociais em saúde relacionadas à classe, gênero, discriminações raciais, idade, entre outras.

Analisa-se neste artigo a assistência ao planejamento reprodutivo em uma clínica da família de um Teias, tendo como referência teórico-analítica os princípios diretivos da Atenção Básica - continuidade, coordenação e integração de cuidados - e o atributo 
da assistência de promoção da autonomia dos usuários nas decisões referentes à vida reprodutiva. Corroborando a proposta de melhoria de qualidade da Atenção Básica, este estudo traz uma análise qualitativa da assistência ao planejamento reprodutivo, agregando ao escopo da avaliação as dimensões dos sujeitos que recebem cuidados e daqueles que estes cuidam. Esta abordagem contribui na análise da assistência ao planejamento reprodutivo na Atenção Básica, visto que as questões subjetivas relacionadas à sexualidade e reprodução são fundamentais no processo de cuidado nessa área de atenção.

\section{Metodologia}

Realizou-se um estudo de caso com abordagem qualitativa em uma clínica da família de um Teias, situado na periferia do município do Rio de Janeiro, utilizando métodos observacionais e análise de relatos orais. $\mathrm{O}$ cenário de estudo foi uma área de grande vulnerabilidade econômica e social, com um dos piores Índices de Desenvolvimento Humano (IDH) da cidade, tendo sido selecionada como área prioritária pelo governo federal para o Programa de Aceleração do Crescimento (ENGSTRON FONSECA; LEIMANN, 2012). A clínica da família foi inaugurada, em 2011, com boa infraestrutura, anexa a uma Unidade de Pronto Atendimento (UPA) e próximo a vários equipamentos sociais, como: unidades de assistência social, inclusive a mulheres vítimas de violência, academia popular, escolas, biblioteca pública e espaços de cultura e lazer. Na clínica da família são oferecidos serviços diversos, entre eles: consultas médicas, consulta de enfermagem, coleta de exames, realização de curativos, atividades educativas, aplicação de vacinas e dispensação de medicamentos. Em 2012, essa clínica aderiu ao Programa Nacional de Melhoria do Acesso e da Qualidade da Atenção Básica
(PMAQ-AB), que tem como principal objetivo induzir a ampliação do acesso e a melhoria da qualidade da Atenção Básica, com garantia de um padrão de qualidade comparável nacional, regional e localmente, de maneira a permitir maior transparência e efetividade das ações governamentais direcionadas à Atenção Básica em saúde.

Em visitas regulares à unidade de saúde, foram descritos aspectos de infraestrutura e do processo de assistência ao planejamento reprodutivo e observados grupos educativos de planejamento familiar. Além disso, foram realizadas 31 entrevistas individuais, com roteiro semiestruturado, com profissionais de saúde - incluindo médicos, enfermeiros, técnicos de enfermagem e agentes comunitários de saúde - e com 30 usuários, cuja idade variou de 14 a 49 anos, incluindo 5 homens e 25 mulheres. O trabalho de campo foi realizado entre janeiro de 2012 e agosto de 2013.

As entrevistas transcritas na íntegra e os registros do diário de campo se somaram e compuseram o material para a análise temática. No processo analítico, procurou-se conhecer as experiências vividas por usuários e profissionais na assistência ao planejamento reprodutivo e as condições facilitadoras e limites dessa assistência no contexto do Teias. Foram realizadas múltiplas leituras do material da pesquisa até que alcançasse o ponto do "desenho significativo de um quadro", multifacetado, mas possível de visões compreensíveis (ALVES; SILVA, 1992, P. 65).

A partir da análise, várias questões foram reveladas: a relação do usuário com a unidade de saúde, o estabelecimento de vínculo entre cuidador e usuário, o papel da unidade como fonte regular de cuidados em saúde e em planejamento reprodutivo e a dimensão da vivência subjetiva e intersubjetiva dos usuários. Os entrevistados foram identificados com nomes fictícios.

A pesquisa foi submetida e aprovada por Comitê de Ética em Pesquisa (CAAE: $15624413408005269)$. 


\section{Resultados}

\section{A assistência ao planejamento reprodutivo na clínica da família no Teias}

A assistência ao planejamento reprodutivo na clínica da família inclui atividade educativa e consultas clínicas - o aconselhamento não tem uma rotina claramente sistematizada e pode acontecer durante uma visita domiciliar, consulta clínica ou em outro momento de atendimento. A atividade educativa possui uma rotina estabelecida - acontecem com regularidade os 'grupos de Planejamento Familiar', cujos processos e infraestrutura são planejados - sendo divulgada por meio de um banner exposto no corredor de atendimento da unidade e pelos profissionais de saúde através de convites individuais, ou ainda, através de moradores da comunidade.

A participação dos usuários no grupo educativo não depende de agendamento e, em geral, é por livre demanda. As reuniões acontecem no início da tarde, mediadas pela equipe de enfermagem, em uma sala específica para a atividade de grupo, com uma boa infraestrutura e com recurso audiovisual e material educativo disponíveis. Os agentes comunitários sabem da existência do grupo e reconhecem a importância dessa atividade, mas apresentam dificuldades em abordar temas relacionados à sexualidade e à reprodução nas visitas domiciliares, seja por desconhecimento ou por constrangimento de tocar em tais assuntos com a população. Os médicos, por sua vez, valorizam pouco o caráter informativo e educativo dos grupos de planejamento reprodutivo e não estão envolvidos com a atividade, considerando mais como uma exigência em caso de demanda por métodos de longa duração (Dispositivo Intrauterino - DIU) ou definitivos (laqueadura e vasectomia).

O grupo de Planejamento Familiar é descrito pelos usuários, na maioria das vezes, como grupo para quem 'não quer ter mais filhos'. Observa-se um pequeno número de participantes nos grupos - inclusive um dos encontros não aconteceu por falta de usuários. O perfil dos participantes foi: mulheres com média de idade de 30 anos, com mais de 2 filhos, a maioria gestante. $\mathrm{O}$ propósito de participação no grupo, em geral, está relacionado à demanda de realizar laqueadura tubária ou inserir o DIU. A abordagem na atividade educativa é direcionada para a contracepção, ignorando a outra dimensão do planejamento reprodutivo: a concepção. No grupo, as dificuldades com os métodos contraceptivos não são problematizadas, e as orientações fornecidas aos usuários também não deixam claro o fluxo de atendimento da assistência ao planejamento reprodutivo. Esse momento da assistência reproduz um modelo normativo que dita um formato de família, com regras de comportamento, sem promover a discussão da realidade vivenciada pelos usuários e seus projetos de vida. A proposta acaba cumprindo muito mais uma exigência legal - inclusive como apontam alguns profissionais - e deixa escapar o seu potencial de promover a autonomia dos usuários para suas escolhas reprodutivas.

Nas consultas clínicas, acontece a prescrição do método contraceptivo oral ou injetável - 'consultas de saúde da mulher', segundo os profissionais -, momento em que, geralmente, é realizado o exame preventivo e exame clínico das mamas. Em consultas clínicas gerais, não há a abordagem por parte dos profissionais quanto aos métodos contraceptivos e também não está sistematizado na unidade a oferta do contraceptivo de emergência. Entre as usuárias, não é incomum o relato da utilização e troca de método por conta própria, além das queixas de dificuldades com os métodos hormonais que nem sempre são acolhidos ou valorizados no serviço de saúde. "[Eu uso] A pílula, desde a minha primeira gravidez, meu filho já tem 12 anos. Eu tomei por conta própria" (Luíza, usuária, 28 anos).

A unidade tem provisão regular dos métodos contraceptivos hormonais orais 
e injetáveis, DIU, preservativo feminino e masculino. Os profissionais identificam a assistência ao planejamento reprodutivo como uma ação direcionada ao público feminino - principal alvo de cuidados nos serviços - e incluem essa assistência no conjunto de ações de atenção à saúde da mulher. Entretanto, percebe-se que não se incluem as mulheres que ainda não iniciaram sua trajetória reprodutiva, mas têm vida sexual ativa, como as adolescentes e as mulheres no climatério, privilegiando um perfil de $\mathrm{mu}$ lheres com filhos e companheiro.

Do ponto de vista do processo e infraestrutura, a clínica tem uma assistência ao planejamento reprodutivo diferenciada em relação a outras unidades de Atenção Básica. Está disponível na unidade um sistema de interconsultas com o Núcleo de Atenção à Saúde da Família (Nasf), que propicia o atendimento por um especialista juntamente com o médico ou enfermeiro da área de cadastro do usuário. Esse sistema garante a resolução de situações especializadas que cabem à Atenção Básica. Esses recursos são incorporados ao seguimento da assistência e visualizados pelos profissionais como maior garantia da continuidade dos cuidados.

O DIU é um método de boa aceitação entre os profissionais e, com frequência, é ofertado às usuárias, o que pode estar relacionado à oferta regular e à facilidade de indicar, inserir e acompanhar os cuidados dentro da própria unidade (inclusive com ultrassonografia). Para os que desejam o método definitivo - laqueadura tubária ou vasectomia -, a participação no grupo educativo é passagem obrigatória, devendo retornar à consulta clínica para continuar o processo. Apesar de terem disponível um sistema de encaminhamento articulado em rede com outros níveis de assistência - o Sistema de Regulação de Vagas (Sisreg) -, este é desacreditado pelos profissionais, em geral associado à grande demora para realização do procedimento. Não há um acompanhamento desses usuários pela Equipe de Saúde da Família, e, por sua vez, a clínica da família não recebe informações se os usuários foram ou não atendidos no serviço de referência. A falha nessa sequência de cuidados leva a desencontros na assistência, fazendo com que esses usuários desistam de realizar o procedimento.

Continuidade, coordenação e integração de cuidados na assistência ao planejamento reprodutivo

A proximidade geográfica da clínica da família com a comunidade e a organização da assistência tanto para atender consultas programadas como demandas espontâneas facilitaram o acesso e a criação de vínculos com o serviço de saúde. O vínculo institucional parece se sobrepor ao vínculo pessoal com um dado profissional, o que por um lado é reflexo da rotatividade e a falta de profissionais, mas por outro, pode apontar para ganhos, já que a mobilidade de profissionais nesse nível de assistência não configura um desmantelamento no vínculo entre usuários e serviços. A análise do vínculo, segundo alguns grupos de usuários, aponta que os adolescentes não veem a unidade como fonte de cuidados à saúde - o que impõe desafios importantes à construção de vínculos com esse grupo - e que os homens a frequentam pouco, particularmente as ações de planejamento reprodutivo. As explicações dos profissionais sobre a falta de vínculos e de cuidados regulares dos homens com a saúde têm vários vieses de gênero, em especial o papel masculino de 'provedor da família', que não lhe permitiria disponibilidade de tempo para os cuidados em saúde.

Alguns dispositivos assistenciais contribuem para a continuidade dos cuidados prestados aos usuários na clínica de família. Um deles é a busca ativa de usuários que abandonaram tratamento ou pararam o seguimento da assistência. Outro dispositivo importante é a dose oral supervisionada, utilizada para garantir a continuidade de tratamento. Na assistência ao planejamento reprodutivo, esses dispositivos não são acionados nem são sistematizados pelas 
equipes. Um terceiro conjunto de dispositivos importantes para a continuidade dos cuidados são os mecanismos e instrumentos de seguimento e acompanhamento da clientela através de sistema informatizado no qual se insere o prontuário eletrônico. Esse instrumento pode disponibilizar mecanismos de alerta para os cuidados, mas não são adotadas pelas equipes para acompanhamento dos cuidados em planejamento reprodutivo, como no acompanhamento de usuárias de métodos hormonais e os que foram encaminhados para método definitivo. O grupo de Planejamento Familiar é também um dispositivo que favorece a continuidade do cuidado, sendo a porta de entrada para os cuidados em planejamento reprodutivo, mas que também funciona como disparador de uma sequência de cuidados. Em um dos depoimentos, identificou-se esse potencial da atividade educativa que oportunizou cuidados em saúde para uma usuária.

Ele me deu um papelzinho que anota os dias do grupo, falou que eu já tinha assistido, eu voltei à médica, ela marcou a data para mim por o DIU, só que sendo que eu tinha que esperar o preventivo, que eu tinha feito um preventivo, aí quando veio esse preventivo já veio com esse problema, com essa alteração que eu fui para lá pro... [encaminhada para a unidade de referência para o tratamento de patologia cervical]. (Teodora, usuária, 29 anos).

No que tange ao princípio da coordenação de cuidados, perceberam-se fragilidades importantes. O modelo de organização da assistência ainda segue a oferta dos programas, o que é apontado pelos profissionais como impeditivo para a abordagem integral em saúde e ao planejamento reprodutivo.

A realidade, mesmo você sendo muito restrito... ela te atropela. E eu já tive, por exemplo, paciente de tuberculose, por causa do uso do mesmo esquema [...], provavelmente não teve a mesma eficácia com o anticoncepcional usando o método só... e engravidou. Então virou uma coisa dupla, ela terminou o tratamento de tuberculose fazendo o pré-natal. [...] Eu ainda acho a gente muito caixinha entendeu? [...] Assim, eu até tento orientar, [...] eu acho que a gente procura mudar essa lógica, mas eu ainda acho que a gente é muito programático. Que tudo é visto dessa forma, a gente é cobrado dessa forma ainda, infelizmente também. (Lucca, médico).

Nas consultas clínicas, perdem-se oportunidades para coordenação de cuidados e, especialmente, para a atenção ao planejamento reprodutivo. Identificou-se que na assistência às mulheres que fazem tratamento para diabetes e hipertensão não há a sistematização da abordagem quanto ao método contraceptivo utilizado, situação que pode trazer riscos para as usuárias, considerando a associação de métodos hormonais com doenças crônicas (diabetes, doenças cardiovasculares, entre outras).

A integração da Atenção Básica com outros níveis de atenção é fundamental para promover uma assistência integral. Quanto a esse princípio, o Teias apresenta a proposta de gestão da Atenção Básica em rede, que agrega unidades parceiras: Nasf, UPA, centro de saúde, maternidade de referência e convênio com unidade de atenção secundária, por exemplo, para tratamento de patologia cervical. A clínica da família dispõe também da rede de serviços especializados do município e estado, acionada através de encaminhamentos que passam pelo Sisreg. A integração da clínica nessa rede organizada potencializa a assistência ao planejamento reprodutivo, facilitando o acesso e ofertando uma atenção resolutiva. A clínica da família tem a possibilidade de resolver um conjunto de demandas reprodutivas maior que outras unidades de Atenção Básica, em especial por ter acesso a uma rede de serviços que não se limita a encaminhar para outros níveis de assistência e traz a gerência de alguns cuidados 
para o nível local, por exemplo, a inserção e o seguimento do cuidado para as usuárias de DIU.

Depois que eu tive meus filhos eu fiz o preventivo que foi para completar o planejamento, precisava fazer o preventivo pra botar o DIU [...] Foi um mês, que eu fiz a palestra em dezembro, em janeiro botei o DIU. [...] Está sendo ótimo eu fiz um ano agora dia 6 de junho e estou bem. Tenho a próxima revisão em outubro e está tudo correndo o melhor jeito possível. (Carina, usuária, 21 anos).

Outra importante integração com a rede se exibe nos encaminhamentos para laqueadura tubária e vasectomia via Sisreg. Embora haja uma rede formalizada, abre-se uma lacuna nas informações e nos cuidados para esses usuários enquanto aguardam o retorno desse encaminhamento. São homens e mulheres que se consideram 'na fila', mas sem que os profissionais saibam de fato o tempo estimado de espera até o retorno do agendamento. Além disso, quando os usuários conseguem o agendamento, eles referem dificuldades com o trâmite de documentos e exames, o que, sem a oferta de apoio da clínica da família, acaba por desencorajar os usuários em continuar o 'processo' de busca pelo método definitivo.

Além de uma rede externa, estão disponíveis serviços e equipamentos sociais no próprio território, como: escolas, igrejas, associação de moradores, serviço de assistência à mulher vítima de violência e espaços de cultura e lazer. Esses parceiros em potencial não aparecerem como partes integrantes de uma rede que aprimore a atenção à saúde, em especial ao planejamento reprodutivo. Eles são acionados quando se depende de um espaço físico ou de materiais para alguma atividade. Essa parceria é limitada, já que poderia também fortalecer as ações desenvolvidas pela unidade de saúde, divulgando-as ou copromovendo-as.

\section{Discussão}

O referencial teórico analítico ofereceu como eixos cada um dos princípios diretivos da atenção à saúde a fim de identificar as potencialidades e fragilidades da assistência ao planejamento reprodutivo. A unidade estudada, apesar de ser um serviço jovem criado no modelo de uma clínica de família e fazer parte de um Teias, com profissionais com qualificação específica para Atenção Básica, ainda herda algumas das dificuldades antigas e arraigadas do sistema de saúde. Por sua vez, é possível atribuir às próprias potencialidades desse modelo de clínica e de território o fato da unidade ter enfrentado e, até certo ponto, superado alguns dos desafios enfrentados pela Atenção Básica, especialmente na assistência ao planejamento reprodutivo.

A assistência ao planejamento reprodutivo na unidade ainda está organizada de maneira vertical, sendo pouco articulada e coordenada com outras ações programáticas (hipertensão, diabetes, tuberculose) e até mesmo com outras ações de saúde reprodutiva (pré-natal, puerpério, rastreio de câncer cervical). Nesse sentido, não há uma clara ruptura com o que foi observado em serviços de Atenção Básica do modelo tradicional centros e postos de saúde - cuja organização da assistência ao planejamento reprodutivo dificulta as ações integrais, fragmentando os sujeitos, suas necessidades de saúde e os cuidados prestados (MOURA; SILVA, 2006; BONAN ET AL., 2010). O desafio de romper com esse modelo é antigo e remete ao PAISM (Programa de Assistência Integral à Saúde da Mulher), que, lançado em 1983, inovava com a proposta de cuidado integral à saúde da mulher não apenas durante o período gestacional - e apontava para um planejamento reprodutivo que extrapolasse a oferta de contraceptivos. A assistência ofertada em outros momentos da vida da mulher para além do ciclo gravídico puerperal, inclusive uma atenção continuada ao planejamento reprodutivo, tem 
também impacto importante nos indicadores de saúde materna (inclusive, na redução da morbimortalidade materna) (COSTA, 2012). Abrir-se a uma visão que não se limite a fases ou ciclos e que considere as necessidades do indivíduo ao longo da vida é um passo importante para romper com a fragmentação do sujeito, predominante no modelo verticalizado, o que se impõe como um desafio à assistência.

Na clínica da família, a organização da assistência ao planejamento reprodutivo segue as diretrizes do Ministério da Saúde, que preveem atividades de aconselhamento, atividade educativa e consulta clínica (BRASIL, 2010). Entretanto, prevalece uma leitura estreita dessas orientações, colocando-as como um conjunto de ações que devem ser ofertadas e não como um processo de cuidados. Essas ações são oferecidas na unidade estudada quase de forma independente entre si, descoordenada e descontinuamente, sem que se defina uma linha de cuidados que as integre, de modo a garantir uma assistência integral à saúde e aos direitos reprodutivos.

A assistência ao planejamento reprodutivo, como é praticada na unidade, volta-se prioritariamente para um público feminino e tende a ecoar a ideia de responsabilidade da mulher pela reprodução (DINIZ, 2000), pouco considerando a diversidade e a complexidade dos sujeitos na vivência da sua vida reprodutiva. Ao entender o planejamento reprodutivo como 'coisa de mulher', ele passa a se destinar a um público específico: mulheres jovens, casadas, com filhos e com demandas de controle de fecundidade, negligenciando adolescentes, homens, mulheres que não iniciaram a sua trajetória reprodutiva e mulheres no climatério. Moura et al. (2010) também observaram a baixa frequência nos serviços de planejamento familiar de adolescentes, homens e mulheres no climatério, apontando para a importância de ações direcionadas para esses grupos.

O planejamento reprodutivo é muitas vezes, de modo explícito ou subentendido, sinônimo de contracepção. Não apenas não se vê o planejamento reprodutivo como parte inerente de toda e qualquer ação em saúde reprodutiva, como também se excluem as demandas relacionadas à infertilidade e à concepção dessas ações. Outros estudos também apontam para essa lacuna na assistência ao planejamento reprodutivo (HEILBORN ET AL., 2009; MOURA; SILVA, 2006), em que grupos que podem ter demandas específicas são negligenciados - como indivíduos que convivem com HIV/ Aids ou com outras condições especiais -, situação que não é tampouco problematizado nas rotinas do serviço.

Alguns dos entraves crônicos da assistência ao planejamento reprodutivo no SUS, e que têm sido apontados na literatura (OSIS ET AL., 2004; COSTA ET AL., 2006), parecem estar sendo superados na unidade estudada. A oferta limitada de métodos nos serviços de saúde tem sido considerada uma restrição importante à garantia do direito das mulheres à livre escolha (COSTA ET AL., 2006). Entretanto, na unidade, a oferta de métodos contraceptivos, como pílulas, injetáveis, DIU e preservativos masculinos e femininos, tem sido regular. A atividade educativa conta com recursos de infraestrutura, como salas próprias, material educativo e expositivo de boa qualidade, e é oferecida regularmente, facultando ao usuário a participação livre em três encontros, sem necessidade de agendamento, facilitando o acesso. Diversos estudos têm apontado que deficiências de infraestrutura e pouco planejamento da oferta da atividade educativa constituem um dos entraves da assistência ao planejamento reprodutivo (PEREIRA ET AL., 2007; BONAN ET AL., 2010), uma realidade superada pelo serviço estudado. Outro aspecto positivo é a integração da unidade com outros serviços do território - em especial o Nasf -, o que possibilita a disponibilidade de exames diagnósticos e a atenção de especialista, oferecendo uma assistência mais resolutiva e diferenciada de outras unidades de Atenção Básica que apresentam dificuldades de se 
articular com outros serviços e níveis da assistência. A integração da clínica da família a uma rede de cuidados organizada no território ampliou o leque de ofertas de métodos contraceptivos, garantindo a inserção do DIU e o seguimento do cuidado.

A infraestrutura da clínica da família apresenta potencialidades que nem sempre são exploradas no intuito de melhorar a assistência ao planejamento reprodutivo. A unidade possui sistema informatizado com prontuário eletrônico e mecanismos de alerta que disponibilizam informações e acompanhamento dos cuidados realizados. Esses recursos permitem a continuidade informacional, um elemento-chave para a continuidade do cuidado (STARFIELD, 2002); e os dados acumulados sobre o usuário permitem instrumentalizar a equipe de saúde para a conduta terapêutica e a avaliação dos resultados. Entretanto, os recursos que o sistema informatizado propicia são pouco explorados pelos profissionais para monitorizar cuidados em planejamento reprodutivo.

Uma situação que, a julgar pelos princípios norteadores da Atenção Básica, seria de pronto superada, não é na prática: persiste a falta de continuidade no acompanhamento das usuárias de métodos hormonais de uso contínuo, especialmente as portadoras de comorbidades. A importância do seguimento da assistência em planejamento reprodutivo é um indicador da qualidade dos serviços, e nem sempre a atividade educativa fornece todos os subsídios necessários para a promoção da autonomia dos sujeitos para o manejo da fecundidade (MOURA; SILVA, 2006). Garantir a oferta continuada de métodos pode ser uma conquista, mas que sem o acompanhamento adequado, com escuta aberta às queixas e insatisfações com o método, as usuárias podem ser expostas a situações de risco.

Embora a equipe de saúde seja jovem, e a maioria dos profissionais tenha qualificação em saúde da família, persistem muitas dificuldades, não tanto no plano técnico-assistencial do planejamento reprodutivo, mas no plano cultural. Mitos e preconceitos - como a associação da liberdade de exercício da sexualidade com as doenças sexualmente transmissíveis, o negligenciamento da sexualidade dos adolescentes e de outros sujeitos, como mulheres no climatério traduzem dificuldades dos profissionais em lidar com questões relacionadas à sexualidade. A abordagem tanto na consulta clínica como na atividade educativa limita-se a dimensão do fisiológico, sem espaço para a discussão de sexualidade e direitos. Ao desconsiderar a dimensão da sexualidade e/ou a sua valorização negativa, os profissionais imprimem tabus e preconceitos nas práticas assistenciais, o que se torna entrave para um cuidado integral - as competências dos profissionais devem ir além do conhecimento técnico-biológico, contemplando também a apropriação sobre os direitos reprodutivos e a consciência de cidadania (ÁVILA, 2003). As dinâmicas entabuladas no grupo de Planejamento Familiar e a disponibilidade, mas com pouca divulgação, da contracepção de emergência ilustram essas dificuldades. Na unidade, a atividade educativa de planejamento reprodutivo não promove diálogo em torno de questões relacionadas à sexualidade e à garantia de direitos. Esses espaços coletivos, como sinalizam os estudos, deveriam promover a troca de experiências quanto a vida sexual e reprodutiva (PEREIRA ET AL., 2007), além de fortalecer a capacidade de escolhas dos sujeitos (OSIS ET AL., 2004; DINIZ, 2000). O contraceptivo de emergência é um método fundamental para que os sujeitos tenham assegurado o direito de controlar a sua fecundidade, e não divulgar a sua disponibilidade no serviço demonstra uma importante limitação da assistência à promoção dos direitos reprodutivos (COSTA ET AL., 2008). No período pesquisado, o contraceptivo de emergência estava disponível na unidade continuamente, mas nem todos os profissionais estavam cientes, não havendo um protocolo específico para a assistência à contracepção de emergência. 
A assistência ao planejamento reprodutivo ofertado pela clínica da família é caracterizada por ações isoladas que, às vezes, se articulam como uma sequência de cuidados, em especial para atender demandas de contracepção. Entretanto, quando propôs-se analisar a assistência ao planejamento reprodutivo como parte da garantia dos direitos reprodutivos, percebeu-se a falta de uma visão ampliada dos sujeitos assistidos e de uma lógica distinta da mera oferta de ações. O processo de construção e implementação da integralidade nas práticas de saúde exige uma recusa do reducionismo biológico e a valorização da 'multidimensionalidade' dos sujeitos, englobando dimensões psicológicas, sociais, culturais e espirituais (MATTOS, 2001). A assistência ao planejamento reprodutivo, enquanto ação da Atenção Básica, deveria ser uma atenção longitudinal (STARFIELD, 2002), com o acompanhamento do usuário ao longo do tempo e a responsabilização da equipe de saúde com o cuidado ao usuário, promovendo o reconhecimento da unidade de saúde como fonte habitual de cuidados. Essas qualidades da atenção são relevantes para a assistência ao planejamento reprodutivo, visto que as demandas reprodutivas vão se modificando de acordo com o tempo, a fase da vida e os interesses dos sujeitos.

A conjugação da Estratégia Saúde da Família com o Teias apresentou ganhos significantes para a assistência ao planejamento reprodutivo. Entretanto, para promover ações integradas, coordenadas e continuadas com vistas a fortalecer a autonomia dos sujeitos para suas escolhas na esfera da vida reprodutiva - e, portanto, que coloque o planejamento reprodutivo em uma dimensão mais ampla que a oferta de serviços e no marco da garantia de direitos -, o serviço deve estar aberto a discussões que revisem as práticas culturais e as moralidades institucionais para que sexo, sexualidade, prazer e reprodução sejam positivamente valorizados em um processo de cuidado que englobe o planejamento reprodutivo.

\section{Considerações finais}

A Atenção Básica é a porta de entrada para os cuidados em saúde, e tem sido interesse do SUS investir na qualificação da assistência nesse nível de atenção, o que se percebe com a expansão da Estratégia Saúde da Família no País na última década. O modelo saúde da família traz um olhar mais aproximado para o contexto dos sujeitos, ampliando a compreensão das suas diversas dimensões. É também nesse nível de atenção que a assistência ao planejamento reprodutivo tem predominantemente sido ofertada. A proposta do Teias traz uma composição e gestão diferenciadas da rede de serviços e inova com estratégias para responder a algumas das necessidades de mudanças da Atenção Básica.

$\mathrm{Na}$ prática, os princípios diretivos da Atenção Básica - continuidade, coordenação e integração de cuidados - ainda não se tornaram expressivos o bastante para dar visibilidade à sua importância no cotidiano assistencial ao planejamento reprodutivo. A Atenção Básica tem limitações próprias e persistentes que não se restringem ao planejamento reprodutivo, mas que afetam diretamente a garantia dessa assistência, por exemplo, as deficiências na integração da unidade com outros níveis de assistência para realização dos métodos cirúrgicos. As dificuldades enfrentadas pelos usuários que demandaram métodos definitivos alertam tanto para a necessidade de melhor articulação da unidade com a rede de saúde como para a necessidade de acompanhamento dessa clientela pela Equipe de Saúde da Família. Em contrapartida, a gestão local dos cuidados pela unidade em parceria com Nasf traz uma resolutividade que é um diferencial da unidade, quando comparada com outros modelos de serviços de Atenção Básica. Esse gerenciamento local do cuidado favorece a satisfação das demandas de saúde no próprio território - entre elas a inserção do DIU e o seguimento do cuidado -, evitando que os 
usuários fiquem 'perdidos na rede de saúde'.

A assistência ao planejamento reprodutivo necessita de insumos e infraestrutura para garantir a oferta de cuidados aos usuários, o que na unidade estudada tem sido um ganho importante, entretanto há outros tão importantes quanto esses quando se almeja uma assistência ao planejamento reprodutivo com vistas à promoção dos direitos reprodutivos. O planejamento reprodutivo não é a soma de ações de aconselhamento, atividade educativa e atividade clínica; ele é um processo de cuidado que visa garantir a homens e mulheres o seu direito de decidir quando e quantos filhos querem ter. É preciso que se constitua uma atenção que supere o reducionismo biológico, promova espaços dialógicos com a troca de vivências sobre sexualidade e reprodução e fortaleça a autonomia dos sujeitos para suas escolhas reprodutivas. Nesse sentido, o arcabouço teórico-analítico propiciou compreender que a assistência na unidade estudada ainda sofre a influência dos modelos tradicionais, reproduzindo uma atenção verticalizada, com pouca coordenação de cuidados e com práticas arraigadas por mitos e preconceitos. A proposta de avaliação qualitativa identificou fragilidades da assistência ao planejamento reprodutivo em uma unidade da Atenção Básica que a avaliação normativa talvez não alcançasse. Por se situar no campo do subjetivo, na experiência relacional dos sujeitos com o serviço de saúde, este estudo dá visibilidade a alguns entraves que nem sempre podem ser encontrados através da utilização de metodologias interessadas somente nos dados produzidos pelo serviço.

\section{Referências}

ALVES, Z. M. M. B.; SILVA, M. H. G. F. D. Análise qualitativa de dados de entrevista: uma proposta. Paidéia (Ribeirão Preto), Ribeirão Preto, v. 2, p. 61-69, fev./jul. 1992.

ÁVILA, M. B. Direitos sexuais e reprodutivos: desafios para as políticas de saúde. Cad. Saúde Pública, Rio de Janeiro, v. 19 supl. 2, p. s465-s469, 2003.

BONAN, C. et al. Avaliação da implementação da assistência ao planejamento reprodutivo em três municípios do estado do Rio de Janeiro entre 2005 e 2007. Rev. Bras. Saude Mater. Infant., Recife, n. 10, supl.1, p. s107-s118, nov. 2010.

BRASIL. Ministério da Saúde, Secretaria de Atenção à Saúde, departamento de Atenção Básica. Saúde sexual e saúde reprodutiva. Brasília, DF, Ministério da Saúde, 300 p., 2010. (Caderno de Atenção Básica, n. 26).

Ministério da Saúde. Secretaria de Atenção à

Saúde. Direitos Sexuais e Direitos Reprodutivos: uma prioridade do Governo. Brasília, DF: Ministério da Saúde, 2005.

\begin{abstract}
Ministério da Saúde. Secretaria de Atenção
à Saúde. Departamento de Ações Programáticas Estratégicas. Política Nacional de Atenção Integral à Saúde da Mulher: princípios e diretrizes. Brasília, DF: Ministério da Saúde, 2004.
\end{abstract}

COSTA, A. M. Política de Saúde Integral da Mulher e direitos sexuais e reprodutivos. In: GIOVANELLA, L. et al. Política e sistema de saúde no Brasil, Rio de Janeiro: Fiocruz, 2012, p. 979-1009.

\section{COSTA, A. M.; GUILHEM, D.; SILVER, L. D.}

Planejamento familiar: autonomia das mulheres sob questão. Rev. Bras. Saude Matern. Infant., Recife, v. 6. n. 1, jan./mar. 2006.

COSTA, N. F. P. et al. Acesso à anticoncepção de emergência: velhas barreiras e novas questões. Rev. Bras. Ginecol. Obstet., Rio de Janeiro, v. 30, n. 2, p. 55-60, fev. 2008.

CUNHA, E. M.; GIOVANELLA, L. Longitudinalidade/ continuidade do cuidado: identificando dimensões e variáveis para a avaliação da atenção primária no 
contexto do sistema público de saúde brasileiro. Ciência e Saúde Coletiva, Rio de Janeiro, v. 16, mar., p. 1029-1042, 2011.

DINIZ, S. G. Cuidando do prazer: do planejamento familiar à contracepção e autonomia das mulheres à responsabilidade compartilhada. In: ARAUJO, M. J. O. et al. Saúde das mulheres: experiências e prática do coletivo feminista sexualidade e saúde. São Paulo, coletivo feminista sexualidade saúde, 2000, p. 43-60.

ENGSTROM, E.; FONSECA, Z.; LEIMANN, B. (Org.). A experiência do Território Escola Manguinhos na rede primária de saúde. Rio de Janeiro: ENSP/FIOCRUZ, 2012.

GIOVANELLA, L. et al. Saúde da Família: limites e possibilidades para uma abordagem integral de atenção primária à saúde no Brasil. Rio de Janeiro, Ciência $\mathcal{E}$ Saúde Coletiva, v. 14, n. 3, p. 763-794, 2009.

HEILBORN, M. L. et al. Assistência em contracepção e planejamento reprodutivo na perspectiva de usuárias de três unidades do Sistema Único de Saúde no Estado do Rio de Janeiro, Cad. Saúde Pública, Rio de Janeiro, n. 25 supl. 2, p. S269-S278, 2009.

MATTOS, R. A. Os sentidos da integralidade, algumas reflexões sobre valores que merecem ser defendidos. In: PINHEIRO, R.; MATOS, R. A. Os sentidos a integralidade na atenção e no cuidado à saúde. Rio de Janeiro: IMS:UERJ:ABRASCO, p. 39-66, 2001.
MOURA, E. R. J.; SILVA, R. M. Qualidade da assistência em planejamento familiar na opinião de usuárias do programa Saúde da Família. Acta Paul enferm., Rio de Janeiro, v. 19, n. 2, p. 150-156, abr./jun. 2006.

MOURA, E. J. et al. Perfil demográfico, socioeconômico e de saúde reprodutiva de mulheres atendidas em planejamento familiar no interior do Ceará. Revista Baiana Saúde Pública, Salvador, v. 34, n. 1, p. 119-133, jan./mar. 2010.

OSIS, M. J. D. et al. Escolha de métodos contraceptivos entre usuárias de um serviço público de saúde. Caderno de Saúde Pública, Rio de Janeiro, v. 20, n. 6, p. 15861594, nov./dez. 2004

PEREIRA, Q. L.C. et al. Processo de (re) construção de um grupo de planejamento familiar: uma proposta de educação popular em saúde. Texto contexto-enferm., Florianópolis, v. 16, n. 2, p. 320-325, abr./maio 2007.

STARFIELD, B. Atenção Primária: equilíbrio entre necessidades de saúde, serviços e tecnologia. Brasília: UNESCO, Ministério da Saúde, 2002. 726 p. Disponível em: <http://www.dominiopublico.gov.br/pesquisa/ DetalheObraForm.do?select _action=\&co_obra=14609>. Acesso em: 20 mar. 2014

Recebido para publicação em abril de 2014 Versão final em dezembro de 2014

Conflito de interesse: inexistente

Suporte financeiro: não houve 